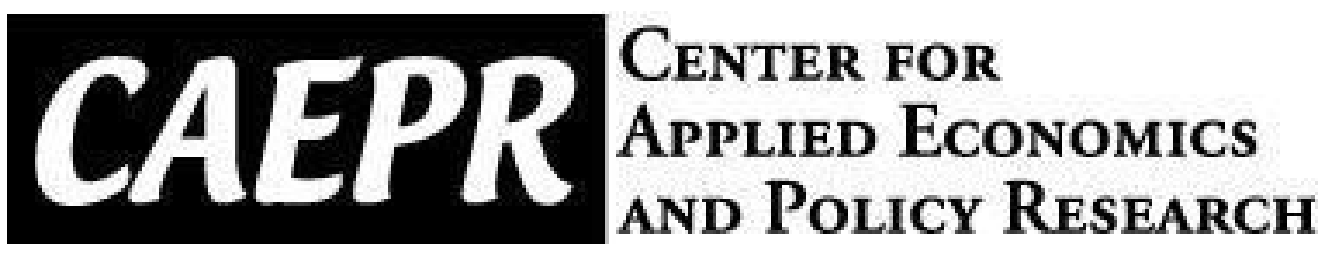

CAEPR Working

Paper

\#2017-005

\title{
An Elementary Exposition of the No Strong Arbitrage Principle for Financial Markets
}

\author{
Robert A. Becker \\ Indiana University
}

June 20, 2017

This paper can be downloaded without charge from the Social Science Research Network electronic library at https://ssrn.com/abstract=2990385

The Center for Applied Economics and Policy Research resides in the Department of Economics at Indiana University Bloomington. CAEPR can be found on the Internet at: http://www.indiana.edu/ caepr. CAEPR can be reached via email at caepr@indiana.edu or via phone at 812-855-4050.

C2017 by Robert A. Becker. All rights reserved. Short sections of text, not to exceed two paragraphs, may be quoted without explicit permission provided that full credit, including (c) notice, is given to the source. 


\title{
An Elementary Exposition of the No Strong Arbitrage Principle for Financial Markets*
}

\author{
Robert A. Becker \\ Department of Economics \\ Wylie Hall \\ Indiana University \\ Bloomington, IN 47405 \\ USA
}

June 20, 2017

Revised August 3, 2017

\begin{abstract}
A Linear Pricing Rule is established for the No Strong Arbitrage Principle (NSAP) in a finite state, single period asset pricing model. The (NSAP) condition is a statement about the inconsistency of a particular system of linear inequalities. The novelty here lies in the use of the Kuhn-Motzkin-Fourier elimination technique that derives the correspionding dual linear inequality system using elementary methods only. The advantage is that a familiar computational scheme yields the relationship between the (NSAP) inequalities and their dual system. Indeed, the method uncovers why the dual inequality system is, in fact, a dual system in the first place. Students and researchers unfamiliar with systems of dual linear inequalities and Theorems of the Alternative may find the approach taken here as a way to better understand the motivation and use of these techiques.

Key Words: No Strong Arbitrage, arbitrage, Farkas Lemma, KuhnMotzkin-Fourier Elimination, state prices, linear inequalities.

JEL Codes: G10, G12, D53, C61.
\end{abstract}

*I thank Frank Page and Todd Walker for comments on a preliminary version of this article. 


\section{Introduction}

The Fundamental Theorem of Asset Pricing connects the absence of arbitrage opportunities in a well-functioning securities market to the existence of a positive linear pricing rule that prices all assets, whether marketed directly, or not. ${ }^{1}$ This Fundamental Theorem is an equivalence claim: there is no arbitrage if and only if there is a positive linear pricing rule. The positive pricing rule is a linear functional on the underlying commodity space of state contingent consumption claims. The basic model assumes a finite number of states-of-nature and a finite number of marketed assets whose market prices are treated as given. The lack of arbitrage opportunities is imposed as a necessary equilibrium condition. The pricing theory so derived is a relative value theory since it depends on the given specification of the contingent payoffs for the unit level of each marketed asset as well as the given market prices per unit for each asset.

The resulting positive linear functional determined by the Fundamental Theorem evaluates contingent claims as the inner product of a vector of state prices and each given contingent claim. The state price vector's $k^{\text {th }}$ - component is interpreted as the price (in some unit of account) paid at time zero for delivery of one unit of consumption goods at time one if and only if state $k$ occurs. The Theorem's linear valuation rule expresses the given price of one unit of each marketed asset as the sum over all states of the product of the asset's contingent payoff in each state times the corresponding state price. In this way the state price vector aggregates the values of claims paid state-by-state to yield the asset's ruling market price. Moreover, this aggregation rule takes a linear form. An asset which pays a nonnegative amount in each state and a positive amount in some state will have a positive linear valuation according to the Fundamental Theorem. This follows since the positivity of the pricing rule means each state's price is strictly positive.

The standard proofs of this equivalence between the absence of arbitrage and existence of a positive linear pricing rule and the existence of state prices usually follows from a separating hyperplane argument or as an application of either the Farkas or Stiemke's Alternatives. ${ }^{2}$

Turunen-Red and Woodland [15] proved a version of this equivalence result based on a non-homogeneous form of Gordon's Alternative for a system of dual linear inequalities. Their method proves the state prices are nonnegative and at least one must be strictly positive, a weaker property than demonstrating all state prices must be positive. Their method applies the Kuhn-MotzkinFourier (KMF) elimination procedure to the system of linear inequalities expressing the absence of arbitrage in a market equilibrium. My exposition unpacks their derivation of the dual system in a case where a particular economic

\footnotetext{
${ }^{1}$ Ross [11] is the seminal paper in this subject. Dybvig and Ross [5] name this result as one that is fundamental. They also refer to it as the Fundamental Theorem of Finance. It is also equivalent to the so-called Representation Theorem. See Ross [12] as well. See LeRoy and Werner [9] for an excellent textbook treatment of this subject.

${ }^{2}$ See Dybvig and Ross ([5], [6]) and Ross [12] for separating hyperlane arguments. See Ross [11] for the Farkas Alternative proof and see Bailey [2], Border [3], or LeRoy and Werner [9] for the Stiemke Alternative argument).
} 
model and equilibrium principle motivates the underlying linear inequalities. This exposition of a special case of the Turunen-Red and Woodland [15] arguments clarifies the way the dual system arises in the absence of strong arbitrage opportunities. ${ }^{3}$

The fact that the absence of arbitrage is expressed via a system of linear inequalities means that the Fundamental Theorem belongs to the subject matter of linear inequalities and hence, a proof using those methods alone is preferred, at least on mathematical grounds. ${ }^{4}$ My paper also follows up on Turunen-Red and Woodland's approach in order to emphasize the state prices can be uncovered using elementary methods only and additional economic insight may be gained in doing so. In particular, the method of elimination finds the dual system of inequalities as a by-product of the elimination procedure rather than identifying an unmotivated dual system in advance as is common in the literature whenever proofs directly invoke Theorems of the Alternative. The basic approach taken here is elementary: Kuhn's [8] elimination method uses only basic arithmetical operations on real or rational numbers. This conforms to Weyl's [18] views on elementary mathematical operations.

There is a significant pedagogical advantage as well to an elementary derivation of the Fundamental Theorem (at least for the form presented here). At many universities undergraduates learn Gaussian elimination in finite mathematics classes, or elementary linear algebra courses. Students at both undergraduate and graduate levels today are rarely exposed to linear inequality systems and Theorems of the Alternative. ${ }^{5}$ Hence, seeing these methods at close range in an interesting application might motivate some students to learn these techniques. First year doctoral economics students might also benefit by studying the geometric interpretation of linear inequality theorems as separation theorems for convex cones. ${ }^{6}$ The general separating hyperplane theorems might seem less mysterious when applied to mathematical programming theory or the standard welfare economics theorems. There are also computational reasons for following Kuhn's [8] elimination ideas in the asset pricing setup. It is known that systems of linear inequalities can be solved in polynomial time when the field of rational numbers expresses all the problem's data (see Border [4] and Shrijver [13]). Hence, it is possible, in principle, to detect whether or not an arbitrage opportunity exists by actually calculating a solution to the relevant inequalities. This point goes beyond the scope of Turunen-Red and Woodland's paper. It is developed further below and in the conclusion.

\footnotetext{
${ }^{3}$ Kuhn [8] derives the dual systems in the proofs of his main results. However, he focuses attention on the elimination procedure's implications for solvability, or unsolvability, of the given inequalties rather than emphasizing the dual system in its own right. Schrijver ([13], p. 89, and pp. 156-157) notes Kuhn effectively obtains a brief induction proof of a variant of the Farkas Alternative from his development of the elimination method.

${ }^{4}$ Weyl [18] emphasizes this perspective on proof methods for linear inequality systems.

${ }^{5}$ This fact has not changed since my undergraduate days a half-century ago.

${ }^{6}$ This geometric perspective is not pursued in the present paper as the algebraic viewpoint is emphasized here instead.
} 


\section{The Asset Market Model}

The asset model is formalized by a pair of objects: $(G, p)$, the market data. $G$ is an $m \times n$ matrix; it is also written as $G_{m \times n}$ when it is important to distinguish its dimension. Risk is modeled by assuming investors act on their asset sales and purchases at time zero $(t=0)$ and receive their payoffs when one of the mutually exclusive states-of-nature, or simply states, occurs at time one $(t=1)$. There are a finite number of states, $m$, and finitely many assets, denoted by $n$. These assets are assumed to be available for trade in the financial marketplace and will be referred to as the marketed assets. A unit of an asset is an $m$-dimensional vector expressing the payoff of one unit of that asset in each state. Assume there are at least two states and one asset. So, $G_{m \times n}$ is the state-asset payoff matrix where $g_{k j}$ is the payoff in units of account realized by one unit of asset $j$ when state $k$ occurs. The vector space of all $m$-tuples of real numbers, $\mathbb{R}^{m}$, is the commodity space or sometimes known as the payoff space. Interest is usually centered on nonnegative payoffs in each state. Use the notation $w$ for a particular payoff vector.

The vector $p>>\theta$ is the given market price of one unit for each security sold in the marketplace. It is known to traders at time zero. The theory developed for the Fundamental Theorem is a relative asset pricing theory. Hence, the primitives in the model include both the payoff matrix, $G$, and the market price vector. The fact that $p$ is given means that the actual process of trade may be suppressed since price determination for the marketed assets is NOT the main issue here. Rather, the Fundamental Theorem is centered on the implications of a particularly minimalist equilibrium notion: the absence of suitably defined arbitrage opportunities. Applications include pricing derivative securities written on a marketed asset, valuation of portfolios of marketed assets, and the famous Modigliani-Miller Theorem (see, for example, Border [3] and $\operatorname{Ross}([11],[12]))$.

States are indexed by $k=1,2, \ldots, m$ and marketed assets by $j=1,2, \ldots, n$. The entries in the payoff matrix $G$ are drawn from either the real number field, or the subfield of rational numbers. The zero vector is denoted by $\theta$, where $\theta=(0,0, \ldots, 0)_{1 \times l}$ or its $l \times 1$ transpose when the meaning is clear. Here, $l=m$ (or $n$ ) depending on context. Likewise, context matters for distinguishing whether a vector is a row or column vector. When the distinction is important, then a transposed vector or matrix has a superscript $T$ : e.g. $G^{T}$. The matrix $G$ exhibits limited liability provided it is a nonnegative matrix. That is, $g_{k j} \geq 0$ for each $k$ and $j$. The market is complete when $\operatorname{rank}(G)=m$ and $m \leq n$. That is, there at least as many assets as states and the column space of $G$ is the $m$-dimensional contingent claims - commodity space. Note that market completeness implies for a given $w$, there is always a portfolio $x$ such that $G x=w$. In case $m=n$, then $x=G^{-1} w$, where $G^{-1}$ is the inverse matrix of $G$.

Investors are assumed to be greedy. They always prefer additional income in any state, other states incomes or payoffs remaining fixed. This is the minimal behavioral assumption underlying the Fundamental Theorem. Investors cannot 
be satiated when their state claims pay off when uncertainty is resolved at time one. It is also possible investors strictly prefer more wealth at time zero since that permits them to spread that additional time zero wealth across the available asset field before knowing nature's state. Investors act by choosing portfolios from the list of marketed assets. Securities markets are perfect. There are no transactions costs. There are no restrictions on the scale of either long or short positions.

\section{The No Strong Arbitrage Principle}

A portfolio is a vector $x \in \mathbb{R}^{n}$ with one component for each marketed asset. The component $x_{j}$ is a scalar identified with the amount of asset $j$ held in this portfolio. A positive component corresponds to a long position and a negative component represents a short position while a zero component signals the asset is not held in the given portfolio. The state-contingent payoff vector at time one is $G x$ when the portfolio $x$ is chosen. It seems reasonable that a portfolio that pays a nonnegative amount in each state and a positive payoff in at least one state would be valued by our greedy investors. That is, this portfolio should cost investors something. The market should not give away state claims for free. The equilibrium condition imposed on the market data $(G, p)$ formalizes this observation. There are two general ways to model this point. The stronger of the two is used in this paper as it is the simplest one with a direct link to KMF elimination.

A strong arbitrage opportunity is a portfolio, $x$, satisfying:

$$
\begin{aligned}
& p x<0 \\
& G x \geq \theta .
\end{aligned}
$$

This is equivalent to

$$
\begin{aligned}
-p x & >0 \\
G x & \geq \theta .
\end{aligned}
$$

Here, vector inequalities are determined by the following considerations: suppose $z \in \mathbb{R}^{l}$. Then

- [nonnegative] $z \geq \theta$ means $z_{i} \geq 0$ for each index $i=1,2, \ldots, l$.

- [semipositive] $z>\theta$ or $z \geq \theta, \neq$, means each $z_{i} \geq 0$ and at least one inequality is strict.

- $\left[\right.$ positive] $z>>\theta$ means each $z_{i}>0$.

The basic equilibrium condition is expressed as a joint restriction on the marketed data. 
The No Strong Arbitrage Principle $(N S A P)$ states for a given market data $(G, p)$ that no strong arbitrage opportunity exists. ${ }^{7}$

$(N)$ is a system of linear inequalities. The set of portfolios satisfying the inequalities of this system is denoted by $S(N)$ and this set may be empty. Indeed, if $(N S A P)$ holds, then $S(N)=\varnothing$, the empty set. The system $(N)$ is solvable if $S(N) \neq \varnothing$ and it is not solvable, or unsolvable, whenever $S(N)=\varnothing$.

The following two theorems form a version of the Fundamental Theorem of Asset Pricing restricted to the case where No Strong Arbitrage opportunity exists. Both theorems maintain the market data, $(G, p)$ as given with $p>>\theta$.

Theorem 1 If (NSAP) holds for the market data $(G, p)$, then there is a semipositive vector $q \in \mathbb{R}^{m}$ such that

$$
q G=p,
$$

or, equivalently, $G^{T} q^{T}=p^{T}$.

Theorem 2 If there is a semipositive vector $q \in \mathbb{R}^{m}$ such that $q G=p$ for the given market data $(G, p)$, then the system $(N)$ is unsolvable and the equilibrium condition (NSAP) holds.

System $\left(N^{*}\right)$ with $q>\theta$ is said to be dual to $(N)$ or that $\left(N^{*}\right)$ is the dual system belonging to $(N)$.

There are two popular ways of proving these results: apply the Farkas Alternative directly to system $(N)$ and its dual $\left(N^{*}\right)$, or a general separation theorem for convex sets. ${ }^{8}$ The former method is the most reasonable one since the question of whether or not there is a strong arbitrage opportunity in the first place belongs to the theory of linear inequalities. In principle, Kuhn-MotzkinFourier elimination can apply as an appropriate proof technique, although this algorithmic method is numerically inefficient. Indeed, it suffers from a curse of "dimensionality." In fact, it is known that the KMF procedure is not a polynomial time algorithm! ${ }^{9}$

The Farkas Alternative approach masks why the systems $(N)$ and $\left(N^{*}\right)$ form dual systems of linear inequalities. Moreover, the elementary approach taken here shows that casting the $(N S A P)$ condition in terms the solvability, or nonsolvability, of a particular system of linear inequalities leads to a natural expression in terms of dual linear systems which form alternatives in the sense that if one of the dual pairs has a solution, then the other does not. A proof working from first principles on the system of linear inequalities $(N)$ uncovers

\footnotetext{
${ }^{7}$ The $(N S A P)$ condition restricts the notion of arbitrage opportunity. The weaker No Arbitrage Principle (NAP) also excludes self-financed arbitrage opportunities of the form $p x=0$ and $G x>\theta$, which do not violate the $(N S A P)$ hypothesis.

${ }^{8}$ The Farkas Alternative or Theorem of the Alternative states that either inequality system $(N)$ has a solution, or system $\left(N^{*}\right)$ has a solution. There is never a solution for both systems.

${ }^{9}$ See Scrijver ([13], p. 156). More background on the historical development of the KMF algorithm can also be found in that text's extensive notes.
} 
this duality relation. I intend to show how this comes about. The basic approach employs only elementary results on the solutions for linear inequalities presented by Kuhn (1956). Recall that elementary means using only basic arithmetical operations on real or rational numbers.

Theorems 1 and 2 have equivalent statements in terms of a primal-dual linear programming pair. See Ingersoll ([7]) and Varian ([16]) for details. ${ }^{10}$ This implies that the simplex method (or, an interior point method) can settle whether or not $(N)$ has a solution. ${ }^{11}$ The simplex method is known to be an excellent algorithm in practice with large problems. ${ }^{12}$ This algorithm stresses pivoting and tableaus as the mechanic for solving linear systems of inequalities. A by-product of solving the primal-dual linear programs is that it is possible to detect whether or not the $(N S A P)$ condition obtains with the given market data. Moreover, this existence issue is solved in a finite number of steps using the simplex method. This is also possible using KMF elimination, although it is impractical when the numbers of assets and states are large. Binomial model examples are presented in Section 6 that can be readily computed by elimination methods. An advantage of the approach taken here is verification of whether or not the system $(N)$ has a solution connects to Guassian elimination. Indeed, this well-known elementary technique readily applies to show whether or not the dual system $\left(N^{*}\right)$ has a solution without resorting to the implementation of a linear programming problem. ${ }^{13}$

One last comment on the computational side: suppose the matrix $G$ and price vector $p$ are given as rational numbers. Then, as long as the elimination steps employ rational numbers only, all computations produce rational outputs. Hence, it is possible to confine attention to the rational numbers, which is an ordered subfield of the real numbers! The formal arguments given below apply to manipulations of the the dual linear inequalities derived from the market data and portfolio weights that are expressed entirely in terms of rational numbers,

\footnotetext{
${ }^{10}$ The primal problem is to choose $x$ to minimize $p x$ subject to the condition $G x \geq \theta$. The corresponding dual problem is to maximize $q \theta$ by choice of $q$ subject to $q G=p$ and $q \geq \theta$. Both authors employ the Duality Theorem of Linear Programming to demonstrate the Fundamental Theorem for the (NSAP) model.

${ }^{11}$ In general, it is known that if rational numbers alone specify the data $(G, p)$, then it is possible to determine in polynomial time whether or not system $(N)$ has a solution. This result applies to both $(N)$ or its linear progamming analog. This is the content of Khachiyan's Theorem based on the ellipsoid method. See Schijver ([13], p. 170) for details. This method is not generally regarded as an efficient way to compute solutions to linear programs or systems of inequalities despite its attractive theoretical properties.

${ }^{12}$ The simplex method is known in artificial examples to visit each possible feasible vertex. It is also known that particular implementations, such as the simplex method with either Dantzig's or Bland's Rules, are not polynomial time algorithms. These tie-breaking Rules are designed to prevent the algorithm from cycling back to a previously encountered basic feasible solution and thereby cycle endlessly without finding the problem's solution. See Schrijver ([13], Chapter 11) for details.

${ }^{13}$ Avis and Klauzny [1] use pivoting and a version of Bland's rule to solve linear inequality systems and prove a variant of the Farkas Alternative while by-passing the related linear programming problem. Also see the discussion in Matoušek and Gärtner ([10], Chapter 6). My approach is less general as it takes advantage of the particular economic structure embedded in the $(N S A P)$ condition.
} 
or the much larger class of real numbers. Of course, real financial market measurements of prices and quantities are confined to rational numbers with finite decimal expansions.

\section{Linear Inequalities and KMF Elimination}

The proofs of Theorems 1 and 2 take advantage of the particular structure of the system $(N)$. This special case of a more general collection of linear inequalities streamlines the application of the KMF algorithm to demonstrate Theorem 1. Kuhn's Rules [8] supporting the elimination algorithm and theorems are briefly recounted in this section. His Rules are analogous to those governing Gaussian elimination, but are carefully drawn to respect the inequality structures while undergoing algebraic manipulations.

For expository purposes rewrite the rows of system $(N)$ explicitly as the collection of inequalities:

$$
-\sum_{j=1}^{n} p_{j} x_{j}>0
$$

and

$$
\sum_{j=1}^{n} g_{k j} x_{j} \geq 0,
$$

for $k=1,2, \ldots, m$. Refer to (0) as the top inequality in $(N)$. The inequalities $(k)$ are said to be type $k$ inequalities. Keep this particular setup in mind as the Rules are presented and applied, although they are clearly more general than the application to $(N)$.

Kuhn's Rules [8] are stated below. They are designed to determine whether or not these inequalities possess a solution and, in principle, find a solution when one exists.

- Rule 1. Any inequality (strict $(>)$ or ordinary $(\geq)$ ) still holds if it is multiplied by a positive number throughout.

- Rule 2. Strict inequality ( $>$ ) holds for the sum of two similarly directed inequalities if and only if strict inequality holds in at least one of the summands. Ordinary inequality $(\geq)$ can always be asserted for the sum.

- Rule 3. Strict inequality $(>)$ always implies ordinary inequality $(\geq)$.

These Rules describe the precise ways in which linear combinations of rows in system $(N)$ can be undertaken without changing the sense of any inequality expressed in $(N)$. Note that a positive scalar must be employed whenever the top inequality is to be multiplied. The second rule asserts combining the top inequality and one of type $(k)$ results in a strict inequality. Likewise, ordinary inequality can always be asserted for a linear combination found according to the second rule by relaxing the strict inequality should one occur in the combination. This possibility is known as weakening the strict inequality. In the formal proof 
of Theorems 1 and 2 it will be useful to maintain the strict inequality should it arise by application of Rule 2 .

A linear combination of rows in $(N)$ found using Rules $1-3$ is said to be a legal linear combination of the rows of $(N)$.

A legal linear combination can be found as follows. Let $Q_{0}$ and $Q_{k}$ be two scalars with $Q_{0}>0$ and $Q_{k} \geq 0$. These scalars are known formally as multipliers. A legal linear combination of the top row and row $(k)$ has the form:

$$
-Q_{0} \sum_{j=1}^{n} p_{j} x_{j}+Q_{k} \sum_{j=1}^{n} g_{k j} x_{j}>0
$$

This legal linear combination is the basic building block in Theorem 1's proof.

The previous inequality is a consequence of $(N)$ if it is satisfied by each solution $x \in S(N)$. If $S(N)$ is empty, then $(N)$ is unsolvable and every inequality in the unknown variable $x$ is a consequence of $(N)$.

Stoer and Witzgall ([14], p.9) note in their presentation of the KMF algorithm that the formation of legal linear inequalities is a transitive process. Choose the inequality following $(k)$ and denote it by $(k+1)$. Multiply inequality $(k+1)$ by the multiplier $Q_{k+1} \geq 0$. Add the result to the legal linear combination shown above, which is itself the result of forming three legal linear combinations. ${ }^{14}$ The resulting inequality is also a legal linear combination of the relations of $(N)$ by Rules 1 and $2 .^{15}$ That is, the following is also a legal linear combination of the relations of $(N)$ :

$$
-Q_{0} \sum_{j=1}^{n} p_{j} x_{j}+Q_{k} \sum_{j=1}^{n} g_{k j} x_{j}+Q_{k+1} \sum_{j=1}^{n} g_{(k+1) j} x_{j}>0 .
$$

This notion of transitivity plays a critical role in the proof of Theorem 1.

The system $(N)$ is inconsistent if the inequality

$$
0 x_{1}+0 x_{2}+\cdots+0 x_{n}>0
$$

is a consequence of $(N)$. Otherwise, system $(N)$ is said to be consistent.

Kuhn [8] proves three theorems. They are combined here as a single series of results applied to $(N)$.

Kuhn's Theorem [8]:

(I) System $(N)$ is solvable if and only if it is consistent.

(II) [A] Every legal linear combination of the inequalities of $(N)$ is a consequence of $(N)$.

[B] If $(N)$ is solvable, then every consequence of $(N)$ is a legal linear combination of the relations of $(N)$.

\footnotetext{
${ }^{14}$ Once for each scalar multiplication by Rule 1 and then the application of Rule 2 for the sum of those first two legal linear combinations.

${ }^{15}$ In the case where $k=m$, then choose $k=1$ as the next relation for this construction.
} 
[C] If $(N)$ is not solvable, then the inequality

$$
0 x_{1}+0 x_{2}+\cdots+0 x_{n}>0
$$

is a legal linear combination of the relations of $(N)$.

(III) The process of elimination either yields a solution for $(N)$ or exhibits the inequality $0 x_{1}+0 x_{2}+\cdots+0 x_{n}>0$ as a legal linear combination of the inequalities of $(N)$. In this case $(N)$ is inconsistent.

The process of elimination is the KMF algorithm. Binomial model examples (Section 6) illustrate the elimination procedure. The mechanics of the elimination procedure appear in Kuhn's paper when legal linear combinations of the relations of $(N)$ are formed and further manipulated in his formal proofs.

Theorem 1 turns on Kuhn's Theorem, Part (IIC). ( NSAP) implies $(N)$ is not solvable, so $0 x_{1}+0 x_{2}+\cdots+0 x_{n}>0$ must obtain. The Rules provide a means to connect the existence of state prices to this condition for inconsistency of the relations in $(N) .{ }^{16}$

\section{Proofs of the Theorems}

The formal proofs of the theorems are worked out below.

Proof. (Theorem 1). (NSAP) implies that system $(N)$ is unsolvable. Hence, it is inconsistent by Kuhn's Theorem $(I)$. It follows that for each $x$ the inequality

$$
0 x_{1}+0 x_{2}+\cdots 0 x_{n}>0
$$

is a consequence of $(N)$. The proof (below) shows how to find this consequence as a legal linear inequality of rows of $(N)$, as promised by Kuhn's Theorem $(I I C)$.

Kuhn's Rules 1 and 2 applied to $(N)$ yield legal linear combinations of the system's rows. Choose any portfolio, $x$, and keep it fixed for now. Set $Q_{0}>0$ and let $Q_{k} \geq 0$. Multiply the top row of $(N)$ by $Q_{0}$ and multiply $Q_{k}$ times each element in row $k$ (of the system $G x \geq \theta$ ). Add the two resulting inequalities to obtain:

$$
-Q_{0} \sum_{j=1}^{n} p_{j} x_{j}+Q_{k} \sum_{j=1}^{n} g_{k j} x_{j}>0 .
$$

As previously observed, this is a legal linear combination of these two rows according to Kuhn's Rules. Next, observe this inequality is also a consequence of $(N)$ since it must hold for any $x$ as there are, by assumption, no solutions to system $(N)$.

\footnotetext{
${ }^{16}$ Kuhn's theory formally includes an inequality of the form $0 x_{1}+0 x_{2}+\cdots 0 x_{n}>-1$, which may be weakened to $0 x_{1}+0 x_{2}+\cdots 0 x_{n} \geq-1$. The inequality $-p_{1} x_{1}-\cdots-p_{n} x_{n}>0$ is, in fact, also a legal linear combination of the inequalities $0 x_{1}+0 x_{2}+\cdots 0 x_{n} \geq-1$ and $-p_{1} x_{1}-\cdots-p_{n} x_{n}>0$. This formal requirement is required by Kuhn ([8], p. 224) to place a standard contradiction (see below text) of the form (1) within reach of the KMF elimination procedure.
} 
If the derivation of (2) is repeated for each $k, k=1,2, \ldots, m$, and summed over all $k$ with multipliers $Q_{k} \geq 0(k=1,2, \ldots, m)$, then transitivity for legal linear combination processes implies the following inequality is a legal linear combination of the rows of system $(N)$ :

$$
-Q_{0}\left(\sum_{j=1}^{n} p_{j} x_{j}\right)+\sum_{k=1}^{m} Q_{k} \sum_{j=1}^{n} g_{k j} x_{j}>0 .
$$

Since $Q_{0}>0$, the next inequality obtains with $q_{k}=Q_{k} / Q_{0} \geq 0$ for $k=$ $1,2, \ldots, m$ :

$$
-\left(\sum_{j=1}^{n} p_{j} x_{j}\right)+\sum_{k=1}^{m} q_{k}\left[\sum_{j=1}^{n} g_{k j} x_{j}\right]>0,
$$

or rewriting it as

$$
-\left(\sum_{j=1}^{n} p_{j} x_{j}\right)+\sum_{k=1}^{m} \sum_{j=1}^{n} q_{k} g_{k j} x_{j}>0 .
$$

Hence, interchanging the order of the double sum above leads to:

$$
-\left(\sum_{j=1}^{n} p_{j} x_{j}\right)+\sum_{j=1}^{n}\left[\sum_{k=1}^{m} q_{k} g_{k j}\right] x_{j}>0 .
$$

Rearrangement produces the inequality:

$$
\sum_{j=1}^{n}\left[-p_{j}+\sum_{k=1}^{m} q_{k} g_{k j}\right] x_{j}>0 .
$$

This is also a legal linear combination of the rows of the system $(N)$. This system can be rewritten in matrix notation as

$$
(p-q G) x>0 .
$$

The condition (NSAP) and Kuhn's Theorem (IIC) imply (1) holds as a legal linear combination of the inequalities of $(N)$. Hence, there must exist a choice of the nonnegative multipliers $q_{k}, k=1,2, \ldots, m$, such that

$$
q G=p,
$$

which is none other than the dual system $\left(N^{*}\right)$. Therefore $(N)$ is unsolvable, and thus inconsistent, implies there is a nonnegative vector $q=\left(q_{1}, q_{2}, \ldots, m\right)$ such that

$$
-p_{j}+\sum_{k=1}^{m} q_{k} g_{k j}=0
$$


holds for each asset $j$. Therefore if condition (1) holds, then the dual system $\left(N^{*}\right)$ has a nonnegative solution. In fact, $p>>\theta$ implies that any nonnegative solution to $\left(N^{*}\right)$ must be semipositive as well. ${ }^{17}$

The proof of the converse result is next.

Proof. (Theorem 2). Suppose there exists a semipositive vector $q$ such that $q G=p$. Then, for any portfolio vector $x$ (including $x=\theta$ ):

$$
q G x=p x,
$$

or

$$
(-p+q G) x=0 .
$$

Suppose that $(N)$ has a nonempty solution set, $S(N)$. That is, $(N)$ is consistent. Then, in particular, each $x \in S(N)$ must satisfy equation (6). Hence, for any $x \in S(N)$, the legal linear combination of the rows of $(N)$ with multipliers $Q_{0}=1, Q_{k}=q_{k}$ for $(k=1,2, \ldots, m)$ expressed by

$$
(-p x)+q G x>0
$$

must be a consequence of $(N)$ as well. The obvious rearrangement of (7) is:

$$
(-p+q G) x>0 .
$$

It is also a consequence of $(N)$. But $-p+q G=\theta$ by hypothesis. Therefore, (8) implies for each $x \in S(N)$ that

$$
0 x_{1}+0 x_{2}+\cdots+0 x_{n}>0
$$

is a consequence of $(N)$, which is impossible if the system $(N)$ is consistent. Hence, it must be the case that there are NO solutions to $(N)$ and the (NSAP) equilibrium condition holds for system $(N)$.

\section{Binomial Market Examples}

Binomial asset markets are a natural source for examples illustrating Theorems 1 and 2. A binomial market model has two states-of-nature denoted as states 1 and 2, respectively. The basic model also assumes two marketed assets. The payoff matrix is $2 \times 2$ and exhibits limited liability. Three examples are noted and use the following payoff matrix:

$$
G=\left[\begin{array}{rr}
60 & 55 \\
40 & 55
\end{array}\right]
$$

Clearly asset 1 is risky and asset 2 is safe - it pays the same in each state. These two marketed assets form a complete market.

\footnotetext{
${ }^{17}$ This follows from the economic structure of $(N)$ with $p>>$. Clearly $q=\theta$ contradicts $p>>\theta$. This conclusion might not follow if $p$ is merely semipositive.
} 
The first example shows how KMF elimination can, in principle, be used to prove the No Strong Arbitrage Opportunity assumption holds for a given inequality system of type $(N)$. Of course, this example is illustrative of the computations involved simply because there are just two states and two assets.

$\star$ Example 1: The price vector is $p=(50,50)$. I claim that $(G, p)$ has no strong arbitrage opportunities. The point of this example is to apply KMF elimination to the system $\left(N_{1}\right)$ :

$$
\begin{aligned}
-50 x_{1}-50 x_{2} & >0 \\
60 x_{1}+55 x_{2} & \geq 0 \\
40 x_{1}+55 x_{2} & \geq 0 .
\end{aligned}
$$

Here $x=\left(x_{1}, x_{2}\right)$ is a portfolio (column) vector. Clearly $x=\theta$ cannot satisfy this system. Claim: $S\left(N_{1}\right)=\varnothing$. Use Kuhn's Rules to verify this observation. I combine some steps in organizing the elimination procedure. Begin by dividing the top inequality by $(1 / 50)$, then divide the second one by $(1 / 60)$ and follow this up by dividing the last one by $(1 / 40)$ to obtain the system $\left(N_{11}\right)$ :

$$
\begin{aligned}
-x_{1}-x_{2} & >0 \\
x_{1}+\frac{11}{12} x_{2} & \geq 0 \\
x_{1}+\frac{11}{8} x_{2} & \geq 0 .
\end{aligned}
$$

This has the same solution set $S\left(N_{1}\right)$ since the legal linear combinations yielding $\left(N_{11}\right)$ are consequences of the relations of $\left(N_{1}\right)$. Next, find legal linear combinations to eliminate $x_{1}$. Note, this top inequality must always have a positive multiplier. Here, let 1 be that multiplier, and add this row to the second row to obtain:

$$
\begin{aligned}
-x_{1}-x_{2} & >0 \\
-\frac{1}{12} x_{2} & >0 \\
x_{1}+\frac{11}{8} x_{2} & \geq 0 .
\end{aligned}
$$

Notice that the second row is now a strict inequality according to Kuhn's Rules. This system also has the solution set $S\left(N_{1}\right)$. Repeat this for the third row and obtain:

$$
\begin{aligned}
-x_{1}-x_{2} & >0 \\
-\frac{1}{12} x_{2} & >0 \\
+\frac{3}{8} x_{2} & >0 .
\end{aligned}
$$

Clearly $S\left(N_{13}\right)=S\left(N_{1}\right)$ as before. It is easy to confirm that no pair $x_{1}$ and $x_{2}$ satisfy this last relation. Hence, there is no portfolio satisfying $\left(N_{13}\right)$. 
As $S\left(N_{13}\right)=S\left(N_{1}\right)$, it follows that $S\left(N_{1}\right)=\varnothing$. Hence, $(G, p)$ satisfies the $(N S A P)$.

The next example finds the corresponding state prices based on the absence of strong arbitrage opportunities.

$\star$ Example 2: Let $(G, p)$ be given from Example 1. The system $\left(N_{1}\right)$ is inconsistent as $S(N)=\varnothing$ :

$$
0 x_{1}+0 x_{2}>0
$$

is a legal linear combination of the rows of $\left(N_{1}\right)$ for any choice of $x$ and Theorem 1 applies. Follow the Theorem 1's proof to show that the vector $q^{*}=\left(\frac{15}{22}, \frac{5}{22}\right)$ satisfies $q^{*} G=p$. This complete market example yields a state price vector uniquely determined by the market data. Moreover, the state price vector is positive, not merely semipositive.

Theorem 2 can be used to see if the dual system has a solution or not using Gaussian elimination to solve a system of linear equations. In Example 2 there is a solution and it is also strictly positive. This is what should be expected as $(N S A P)$ holds for the given complete market's data. The next example shows how to use Theorem 2 to detect the existence of a strong arbitrage opportunity.

$\star$ Example 3: Maintain the matrix $G$ but reset the price vector to $p=$ $(25,50)$. The same approach as taken in Example 1 shows the system $\left(N_{2}\right)$ has at least one solution (check that $x=\left(\frac{8}{5},-1\right)$ works):

$$
\begin{aligned}
-25 x_{1}-50 x_{2} & >0 \\
60 x_{1}+55 x_{2} & \geq 0 \\
40 x_{1}+55 x_{2} & \geq 0 .
\end{aligned}
$$

Hence, there is a strong arbitrage opportunity. Theorem 2 shows this from the dual vantage. Since $G$ is invertible, the system of linear equations $q G=p$ always has a unique solution. In this case,

$$
q^{*}=\left(-\frac{5}{22}, \frac{13}{22}\right)
$$

This solution fails the nonnegativity test for Theorem 2. Hence, there is NO nonnegative (semipositive) solution to the dual system and therefore, it must be the case that $\left(N_{2}\right)$ has a solution!

\section{Conclusion}

The idea of using KMF elimination to prove the Fundamental Theorem of Asset Pricing is due to Turunen-Red and Woodland [15]. Their setup is more general than the one used here based on the absence of No Strong Arbitrage opportunities. Hence, the theorems presented above are special cases of their results. They rely on Gordan's Theorem of the Alternative whereas the better known Farkas Alternative is implicitly highlighted here. Neither approach is sufficient to deduce that the state price vector is positive. Their version of the 
Fundamental Theorem proves state prices form a semipositive vector or pricing rule. A proof of the Fundamental Theorem in the general $(N A P)$ case, based on Stiemke's Alternative, overcomes this limitation and yields a positive linear pricing rule. Stoer and Witzgall ([14], p 24) show Stiemke's Alternative is a corollary to the KMF algorithm. Hence, a KMF elimination argument for the general Fundamental Theorem may be available. This would likely require a direct proof of the Stiemke Alternative by KMF elimination methods for the $(N A P)$ inequalities. ${ }^{18}$

One advantage of my more restricted approach is that the ways in which the dual system emerges seems a bit more organic - that is, derived from the internal logic of the $(N S A P)$ condition coupled with the use of Kuhn's results. Indeed, this approach is available to readers only familiar with Gaussian elimination!

Theorems 1 and 2 contain a hidden existence theorem that settles directly whether or not system $(N)$ has a solution. This is the content of Kuhn's Theorem (III). Of course, Schrijver's [13] monograph informs us that the KMF algorithm is hardly an efficient way to resolve this issue. An alternative strategy offers an indirect existence result: the dual system can also be solved and the converse result tells that IF the dual's solution exists, and at least one state price is negative, then system $(N)$ must have a solution - a strong arbitrage opportunity exists! Gaussian elimination can be used here, but so can any alternative method for solving systems of linear equations which may be more satisfactory from a running time perspective. Of course, the dual system's linear equations form a non-homogeneous system which may have no solution, a unique solution, or many solutions. In the first case conclude there is a strong arbitrage opportunity available. In the third case there is no strong arbitrage opportunity if there is SOME semipositive solution to the dual equations, and in the second case there is no strong arbitrage if and only if the dual's unique solution is semipositive.

The standard proofs of Theorems 1 and 2 utilize either a Theorem of the Alternative (an inequalities based argument) or one of the many standard separating hyperplane theorems which are NOT part of the theory of linear inequalities. ${ }^{19}$ However, Theorems of the Alternative are also readily seen as separating hyperplane theorems when expressed in geometric language. The arguments given in this article seem to circumvent the use of a separation argument to prove Theorem 1. This is an illusion. The separation technique is still there, just heavily disguised via the elimination argument underneath Kuhn's results. The real work of replacing a formal separation argument is buried in the KMF computational algorithm! A constructive argument underlies the assertions in the Theorems 1 and 2, even though the proofs do not require the explicit computations be carried out. All that is needed is to realize

\footnotetext{
${ }^{18} \mathrm{~A}$ proof along these lines would necessarily examine many special cases owing to the less restrictive conditions for no arbitrage portfolio's existence.

${ }^{19}$ Ross [11] states and proves Theorems 1 and 2 when a general no arbitrage condition obtains employing the Farkas Alternatiive. His proof formally yields semipositive state prices. An ad hoc argument is offered to show those semipositve state prices must be positive.
} 
when (4) obtains as a legal linear combination of the rows constituting system $(N)$ and (5) follows.

It is interesting to note that Webster ([17]) develops the theory of linear inequalities based on a separation argument combined with theorems concerning legal linear combinations of relations in the given system. This is a hybrid approach. He takes this short-cut to avoid elementary elimination arguments. Thus, he goes outside the elementary level sought in this note.

\section{References}

[1] David Avis and Bohdan Kaluzny, "Solving Inequalities and Proving Farkas's Lemma Made Easy," American Mathematical Monthly, Vol,. 111 No. 2 (Feb. 2004), pp. 152-157.

[2] Roy C. Bailey, The Economics of Financial Markets, Cambridge University Press, 2005.

[3] Kim C. Border, "Elementary Asset Pricing," Cal Tech Lecture Notes, 1984, updated 1996 and 2002.

[4] Kim C. Border, "Alternative Linear Inequalities," Cal Tech Lecture Notes, September 2013.

[5] Philip H. Dybvig and Stephen A. Ross, "Arbitrage," in Steven F. Durlauf and Lawrence E. Blume, eds., The New Palgrave: A Dictionary of Economics, Volume 1, Second Edition, Palgrave MacMillan, New York, 2008.

[6] Philip H. Dybvig and Stephen A. Ross, "Aribtrage, State Prices and Portfolio Theory," in George M. Constantinides, Milton Harris, and René M. Stulz, eds., Handbook of the Economics of Finance, Volume 1B, Chapter 10 (pp. 605-638) Elsevier North-Holland, Amsterdam, 2003.

[7] Jonathan Ingersoll, Jr., Theory of Financial Decision Making, Rowman \& Littlefield, Totawa, NJ, 1987.

[8] H.W. Kuhn, "Solvability and Consistency for Linear Equations and Inequalities," The American Mathematical Monthly, Vol. 63, No. 4 (Apr., 1956), pp. 217-232.

[9] Stephen F. LeRoy and Jan Werner, Principals of Financial Economics, Second Edition, Cambridge University Press, Cambridge, UK, 2014.

[10] Jiří Matoušek and Bernd Gärdner, Understanding and Using Linear Programming, Springer, Berlin, 2007.

[11] Stephen A. Ross, "Return, Risk, and Arbitrage," in Risk and Return in Finance, Volume I, Irwin Friend and James L. Bicksler, eds., Ballinger Publishing Company, Cambridge, Mass., 1977 (pp. 189-218). 
[12] Stephen A. Ross, Neoclassical Finance, Princeton University Press, Princeton, NJ, 2005.

[13] Alexander Schrijver, Theory of Linear and Integer Programming, John Wiley \& Sons, NY, 1986, paperback edition, 1998.

[14] Josef Stoer and Christoph Witzgall, Convexity and Optimization in Finite Dimensions, I, Springer 1970.

[15] Arja H. Turunen-Red and Alan D. Woodland, "On Economic Applications of the Kuhn-Fourier Theorem," in Topics in Mathematical Economics and Game Theory: Essays in Honor of Robert J. Aumann, Myrna H. Wooders, editor, American Mathematical Society, Fields Institute Communications, Volume 23, 1999, pp. 257-276.

[16] Hal R. Varian, "The Arbitrage Principle in Financial Economics," The Journal of Economic Perspectives," Vol. 1, No. 2 (Autumn, 1987), pp. 5572 .

[17] Roger Webster, Convexity, Oxford University Press, Oxford, 1994.

[18] Hermann Weyl, "Elementary Proof of a Minimax Theorem due to von Neumann," in H.W. Kuhn and A.W. Tucker, eds., Contributions to the Theory of Games, Volume I, Annals of Mathematics Studies 24, Princeton University Press, 1950 (pp. 19-25). 